\title{
THE BERKOVITS COMPLEX AND SEMI-FREE EXTENSIONS OF KOSZUL ALGEBRAS
}

\author{
IMMA GÁLVEZ, VASSILY GORBOUNOV, ZAIN SHAIKH, AND ANDREW TONKS
}

\begin{abstract}
In his extension [3] of W. Siegel's ideas on string quantization, N. Berkovits made several observations which deserve further study and development. Indeed, interesting accounts of this work have already appeared in the mathematical literature $[8,15]$ and in a different guise due to Avramov. In this paper we bridge between these three approaches, by providing a complex that is useful in the calculation of some homologies.
\end{abstract}

\section{Contents}

1. Introduction

2. Preliminaries on commutative Koszul algebras

2.1. Koszul Algebras

2.2. Lie Superalgebras

2.3. Deviations

3. Koszul homology and Berkovits complex

3.1. Koszul homology and the algebra of syzygies

3.2. The Berkovits complex

3.3. Minimal models for commutative Koszul algebras

4. A smaller complex

4.1. The Grassmannians $G(2, N)$

4.2. The Complex

4.3. Further generalization

References

\section{INTRODUCTION}

This paper began with an observation of the importance of Koszul duality theory for commutative algebras in the work of Berkovits [3] on string quantization and string/gauge theory duality. In Berkovits' paper the commutative algebra in question is the projective coordinate algebra $S$ of the orthogonal Grassmannian $O G(5,10)$, related to the spinor representation of the group $S O(10, \mathbb{C})$. This is a commutative quadratic algebra, and for such algebras it is an easy consequence of the definition that its Koszul dual is the universal enveloping algebra of a graded Lie superalgebra $L=\bigoplus_{i \geq 1} L_{i}$.

In a very interesting paper Movshev-Schwarz [15] observed that the algebra of syzygies of $S$ is isomorphic to the cohomology $H^{*}\left(L_{\geq 2}, \mathbb{C}\right)$ of the graded Lie superalgebra $L_{\geq 2}=\bigoplus_{i \geq 2} L_{i}$. They proposed a further complex in an attempt to describe an off-shell formulation of $N=1, D=10$ Yang-Mills theory, and posed the question of calculating its homology. Their construction is an iteration of the Koszul homology of a sequence of elements of an algebra, first applied to the algebra $S$ and then applied to its syzygies. Namely, if $A$ is finitely generated and presented as $\mathbb{C}\left[a_{1}, \ldots, a_{n}\right] / I$, the Koszul homology with respect to the sequence $\left\{a_{i}\right\}$ is called the algebra of syzygies of $A$. The generators $\Gamma_{j}$ 
of the ideal $I$ represent syzygies in the lowest degree. The Koszul homology of the algebra of syzygies with respect to the sequence $\left\{\Gamma_{j}\right\}$ is the algebra studied by Movshev-Schwarz.

The same construction has appeared in the study of deviations of a local ring (see [2] and section 2.3 below). These are a sequence of integers that are attached to a local ring, that measure how far it is from being regular or a complete intersection. In calculating them, Avramov constructs complexes that are analogous to Movshev-Schwarz. A consequence of Avramov's work is that if $A$ is Koszul, the Berkovits homology can be described explicitly in terms of the graded Lie superalgebra L. Namely, for an arbitrary finitely generated commutative Koszul algebra, the Berkovits homology is isomorphic to the cohomology $H^{*}\left(L_{\geq 3}, \mathbb{C}\right)$. This result was also shown by Movshev-Schwarz for the case $A=S$. Further, they suggested an algebraic technique to study the Berkovits homology of the algebra $S$ by constructing an equivalent but "smaller" complex. This complex does not seem to appear as an accident; we believe that it is naturally attached to a commutative Koszul algebra. We construct such a "smaller" complex for a Koszul algebra attached to the Plücker embedding of the Grassmannian $G(2,5)$.

At this point we would like to mention a connection with a conjecture made by Avramov: Conjecture $\mathrm{C}_{10}$ of [1] states that if $A$ is not a complete intersection, then the appropriate Lie superalgebra $L$ contains a free nonabelian graded Lie subalgebra. The algebra $S$ above provides an example confirming the conjecture, according to [11, 15]. Indeed, the "smaller" complex allows one to calculate that $H^{2}\left(L_{\geq 3}, \mathbb{C}\right)$ is trivial. Our construction of a "smaller" complex allows us to show that the Lie superalgebra $L_{\geq 3}$ is free for the projective coordinate algebra of $G(2,5)$, providing another confirmation of $\mathrm{C}_{10}$.

The algebra of syzygies of $S$ is in fact non-quadratic, and yet the "smaller" complex still exists. So, we believe that our construction can be extended to a more general case, but requires (to our knowledge) a substantial extension of the notion of Koszulness to non-quadratic algebras.

The paper is organised as follows: in Section 2 we discuss some preliminaries including Koszul algebras and Lie superalgebras; in Section 3.2 we define the Berkovits complex and how it arises as a semi-free extension of the initial algebra. The main theorem of the paper is Theorem 3.11, which shows that for any commutative Koszul algebra, its Berkovits homology is isomorphic to $H^{*}\left(L_{\geq 3}, \mathbb{C}\right)$. It is in Section 4 where we construct the "small" complex and prove that it calculates the Berkovits homology when we have a Koszul algebra attached to the Plücker embedding of the Grassmannian $G(2,5)$ (Corollary 4.8).

The first author was partially supported by Spanish Government grants MTM201015831, MTM2010-20692, MTM2012-38122-C03-01 and MTM2013-42178-P and Catalan Government grants SGR1092-2009 and SGR634-2014, and the fourth author by MTM2010-15831 and MTM2013-42178-P. All the authors visited the Max Planck Institute while working on this project and are grateful to the Institute for excellent working conditions. The authors also thank to L.A. Bokut, A. Losev and M. Movshev for very useful discussions and advice, and A. Conca and S. Iyengar for pointing us to Avramov's commutative algebra constructions in [2]. We are also grateful to the referee for pointing out an error in [8, Theorem 4.4.1], which we initially used to construct the smaller complex for a larger class of algebras. Of course we also are very grateful to Vadim Schechtman with whom this project originally was started and to whom we dedicate it with our best wishes. 


\section{Preliminaries on commutative Koszul algebras}

We fix our ground field $k=\mathbb{C}$. Unless otherwise stated, all algebras are graded $A=\bigoplus_{i \geq 0} A_{i}$ and locally finite dimensional, i.e. $\operatorname{dim} A_{i}<\infty$ for all $i \geq 0$. Further, we assume that $A_{0}=\mathbb{C}$, so that $A=\mathbb{C} \oplus A_{+}$is augmented, with augmentation ideal $A_{+}=\bigoplus_{i>0} A_{i}$.

\subsection{Koszul Algebras.}

Definition 2.1. Let $A$ be a quadratic algebra defined by a presentation $A=T(V) / Q$, where $V$ is a finite dimensional vector space of generators in degree one, and $Q$ is a twosided ideal generated by quadratic elements. The Koszul dual algebra $A^{!}$is the graded algebra defined as

$$
A^{!}=T\left(V^{*}\right) / Q^{\perp}
$$

where $V^{*}$ is concentrated in degree one and $Q^{\perp} \subset V^{*} \otimes V^{*}$ is the annihilator of $Q$.

Clearly $A^{! !}=A$.

The Koszul complex $\left(K(A), d_{A}\right)$ of a quadratic algebra $A$ is defined by the sequence of right $A \otimes A^{!}$-modules

$$
K_{p}(A):=A \otimes\left(A^{!}\right)_{p}^{*} \quad(p \geq 0) .
$$

Here $A$ is a right $A$-module via multiplication and $\left(A^{!}\right)^{*}$ is a right $A^{!}$-module via

$$
(\varphi b)(c):=\varphi(b c), \quad\left(\varphi \in\left(A^{!}\right)^{*}, b, c \in A^{!}\right) .
$$

The differential $d_{A}$ is the action of the identity $\operatorname{id}_{V} \in \operatorname{Hom}(V, V)=V \otimes V^{*} \subset A \otimes A^{!}$. It has degree -1 , and satisfies $d_{A}^{2}=0$ since $A$ is quadratic.

Recall that since $A$ is graded, the algebra $\operatorname{Ext}_{A}(\mathbb{C}, \mathbb{C})$ is bigraded, and decomposes as

$$
\operatorname{Ext}_{A}^{i}(\mathbb{C}, \mathbb{C})=\bigoplus_{j \geq 0} \operatorname{Ext}_{A}^{i j}(\mathbb{C}, \mathbb{C})
$$

where $i$ is the cohomological grading and $j$ is the grading descending from $A$.

Definition 2.2. An augmented algebra $A$ is a Koszul algebra if $\operatorname{Ext}_{A}^{i j}(\mathbb{C}, \mathbb{C})=0$ whenever $i \neq j$.

The following theorem gives some equivalent definitions of a Koszul algebra. A proof may be found, for example, in [16, Chapter 2, Theorem 4.1].

Theorem 2.3. Let $A$ be a quadratic algebra. Then the following are equivalent:

(1) A is Koszul,

(2) $A^{!}$is Koszul,

(3) $\operatorname{Ext}_{A}(\mathbb{C}, \mathbb{C}) \cong A^{!}$as algebras with $\operatorname{Ext}_{A}^{i}(\mathbb{C}, \mathbb{C})=A_{i}^{!}$,

(4) $\left(K(A), d_{A}\right)$ is a resolution of $\mathbb{C}$.

Example 2.4. Consider the symmetric algebra, $S(V)$. It is a quadratic algebra and the ideal of relations $Q$ inside $T(V)$ is precisely $(u \otimes v-v \otimes u \mid u, v \in V)$. The annihilator of $Q$ inside $T\left(V^{*}\right)$ is generated by tensors of the form $u^{*} \otimes v^{*}+v^{*} \otimes u^{*}$ and so $S(V)^{!}=\bigwedge V^{*}$. Therefore the Koszul complex of $S(V)$ has graded components

$$
K_{p}(S(V))=S(V) \otimes \bigwedge^{p} W_{1} \quad(p \geq 0)
$$

where $W_{1}$ is a copy of $V$ inside $\left(S(V)^{!}\right)^{*}$. If $a_{1}, \ldots, a_{n}$ and $\xi_{1}, \ldots, \xi_{n}$ are bases of $V$ and $W_{1}$ respectively, then the differential can be written as

$$
\sum_{i} a_{i} \frac{\partial}{\partial \xi_{i}} .
$$


This makes $K(S(V))$ into a DG algebra over $S(V)$, with the obvious product structure. It is a resolution of $\mathbb{C}$ (see [14, Proposition VII.2.1] for a proof), so $S(V)$ and $S(V)^{!}=\bigwedge V^{*}$ are Koszul algebras.

\subsection{Lie Superalgebras.}

Definition 2.5. A Lie superalgebra over $\mathbb{C}$ is a $\mathbb{Z}_{2}$-graded vector space $L=L_{\overline{0}} \oplus L_{\overline{1}}$ with a map $[\cdot, \cdot]: L \otimes L \rightarrow L$ of $\mathbb{Z}_{2}$-graded spaces, satisfying:

(1) (anti-symmetry) $[x, y]=-(-1)^{|x||y|}[y, x]$ for all homogeneous $x, y \in L$,

(2) (Jacobi identity) $(-1)^{|x||z|}[x,[y, z]]+(-1)^{|y||x|}[y,[z, x]]+(-1)^{|z||y|}[z,[x, y]]=0$ for all homogeneous $x, y, z \in L$.

Here $|x|=i$ when $x \in L_{\bar{i}}$ for $i=0,1$, and an element $x$ in $L_{\overline{0}}$ or $L_{\overline{1}}$ is termed even or odd respectively. We recover the familiar definition of a Lie algebra (over $\mathbb{C}$ ) in the case $L=L_{\overline{0}}$.

A graded Lie superalgebra is a Lie superalgebra L together with a grading compatible with the bracket and supergrading. That is, $L=\bigoplus_{m>1} L_{m}$ such that $\left[L_{i}, L_{j}\right] \subset L_{i+j}$ and $L_{\bar{i}}=\bigoplus_{m \geq 1} L_{2 m-i}$ for $i=0,1$.

Remark 2.6. In the literature a graded Lie superalgebra is sometimes called simply a graded Lie algebra. However, this could also refer to an ordinary Lie algebra $L=L_{\overline{0}}$ with a grading compatible with the bracket. In order to avoid this ambiguity we prefer the terminology as in the definition.

Let $V$ be a vector space with basis $a_{1}, \ldots, a_{n}$ and consider a quadratic commutative algebra $A=T(V) / Q$. Then, $Q=C \oplus I$ where $C$ is the ideal $(u \otimes v-v \otimes u \mid u, v \in V)$ and $I=\left(\Gamma_{1}, \ldots, \Gamma_{m}\right) \subset S^{2}(V)$ so that we can write $A=S(V) / I$. Further, $Q \supseteq C$ implies that $Q^{\perp} \subseteq C^{\perp}=S^{2}\left(V^{*}\right)$ and so $Q^{\perp}$ is generated by certain linear combinations of anti-commutators $\left[a_{i}^{*}, a_{j}^{*}\right]=a_{i}^{*} a_{j}^{*}+a_{j}^{*} a_{i}^{*}$. We can therefore describe the Koszul dual as the universal envelope of a graded Lie superalgebra,

$$
A^{!}=U(L), \quad L=\bigoplus_{m \geq 1} L_{m}=\mathbb{L}\left(V^{*}\right) / J,
$$

where $\mathbb{L}$ is the free Lie superalgebra functor, the space of (odd) generators $V^{*}$ is concentrated in degree 1 , and $J$ is the Lie ideal with the same generators as $Q^{\perp}$ but viewed as linear combinations of supercommutators.

Definition 2.7. For $k \geq 2$, we define the graded Lie superalgebras

$$
L_{\geq k}=\bigoplus_{m \geq k} L_{m}
$$

The interpretation of the algebras $L_{\geq k}$ for $k>2$ was outlined in [3]. In this paper, we concentrate on the case $k=3$.

2.3. Deviations. Consider the Hilbert series of the commutative algebra $A=S(V) / I$

$$
H_{A}(t):=\sum_{r=0}^{\infty} \operatorname{dim}\left(A_{r}\right) t^{r}=\frac{h(t)}{(1-t)^{n}},
$$

where $h(t)$ is a polynomial such that $h(0)=1$. Gauss' cyclotomic identity [7] allows us to expand this rational function into an infinite product

$$
\frac{h(t)}{(1-t)^{n}}=\prod_{s=1}^{\infty}\left(1-t^{s}\right)^{(-1)^{s} \varepsilon_{s}(A)},
$$

where the exponents $\varepsilon_{s}(A)$ are integers known as the deviations of $A$. 
When $A$ is a Koszul algebra, the exponents $\varepsilon_{s}(A)$ give the dimensions of the graded components $L_{s}$ of the Lie superalgebra $L$ (see for example [13]), and in particular we have $\varepsilon_{1}(A)=n$. Indeed, by the Poincaré-Birkhoff-Witt theorem for the universal enveloping algebra of a graded Lie superalgebra, the Hilbert series of $A^{!}=U(L)$ is equal to

$$
H_{A^{!}}(t)=\prod_{s=1}^{\infty}\left(1-(-t)^{s}\right)^{(-1)^{s-1} \operatorname{dim}\left(L_{s}\right)}
$$

and from the relation $H_{A}(t) H_{A^{!}}(-t)=1$ it follows that $\varepsilon_{s}(A)=\operatorname{dim}\left(L_{s}\right)$ in $(4)$. See [16, Section 2.2] for further details, and also Remark 3.10 below.

Dividing by

$$
\prod_{s=1}^{k-1}\left(1-t^{s}\right)^{(-1)^{s} \varepsilon_{s}(A)}
$$

we obtain the Hilbert series of $L_{\geq k}$ as the following infinite product

$$
\prod_{s=k}^{\infty}\left(1-t^{s}\right)^{(-1)^{s} \varepsilon_{s}(A)} .
$$

\section{Koszul homology And Berkovits complex}

3.1. Koszul homology and the algebra of syzygies. Let $A=S(V) / I$ be a commutative quadratic algebra with $\left\{a_{1}, \ldots, a_{n}\right\}$ a basis of $V$.

Definition 3.1. The Koszul homology of $A$ with respect to a sequence of elements $x_{1}, \ldots, x_{k}$ in $A$ is the homology of the complex

$$
A\left[W_{1}\right]:=\left(A \otimes \bigwedge W_{1}, \sum_{i=1}^{k} x_{i} \frac{\partial}{\partial \theta_{i}}\right)
$$

where $A$ has homological degree zero and $W_{1}$ is the vector space spanned by elements $\theta_{1}, \ldots, \theta_{k}$ in homological degree one.

The algebra of syzygies of $A$ is the Koszul homology of $A$ with respect to the sequence $\left\{a_{1}, \ldots, a_{n}\right\}$.

The algebra of syzygies of $A$ is graded finite dimensional by Hilbert's syzygy theorem (c.f. [6, Theorem 1.1]).

Remark 3.2. We make the following trivial remarks:

(1) Suppose $A$ is the symmetric algebra $S(V)$. Then $A^{!}=\bigwedge V^{*}$ and the complex $A\left[W_{1}\right]$ calculating the algebra of syzygies of $A$ is precisely the Koszul complex, compare Example 2.4.

(2) The complex $A\left[W_{1}\right]$ is a DG algebra. The algebra structure is given by

$$
(a \otimes \omega) \cdot(b \otimes \eta)=a b \otimes \omega \wedge \eta,
$$

where $a, b \in A$ and $\omega, \eta \in \bigwedge W_{1}$. By a theorem of Kadeishvili [12], the Koszul homology, and in particular the algebra of syzygies, inherits an $A_{\infty}$-algebra structure, which is unique up to (non-unique) isomorphism. We call this the $A_{\infty}$-minimal model for $A\left[W_{1}\right]$ (the term minimal model will be reserved for something else).

(3) When $A$ is graded, the Koszul homology is bigraded. In the case of Koszul homology with respect to a sequence of generators, the homological grading will be called the order of the syzygies and the sum of the homological grading and the grading on $A$ will be called the degree of the syzygies. 
We observe that $\operatorname{Tor}^{S(V)}(A, \mathbb{C})$ is also calculated by the complex $A\left[W_{1}\right]$, and so coincides with the algebra of syzygies. Indeed, $K(S(V))$ is a resolution of $\mathbb{C}$ by $S(V)$-modules, so $\operatorname{Tor}^{S(V)}(A, \mathbb{C})$ is the homology of the complex

$$
A \otimes_{S(V)} K(S(V))=A \otimes_{S(V)} S(V) \otimes_{\mathbb{C}} \bigwedge W_{1}=A \otimes_{\mathbb{C}} \bigwedge W_{1}
$$

Alternatively, suppose we have a minimal free resolution of $A$,

$$
\cdots \longrightarrow F_{2} \longrightarrow F_{1} \longrightarrow F_{0} \longrightarrow A \longrightarrow 0,
$$

by graded free $S(V)$-modules

$$
F_{p}=\bigoplus_{q} R_{p, q} \otimes S(V) .
$$

Here minimality means that the differential vanishes on tensoring this complex with the trivial $S(V)$-module $\mathbb{C}$, and hence

$$
\operatorname{Tor}_{p}^{S(V)}(A, \mathbb{C})=R_{p}:=\bigoplus_{q} R_{p, q}
$$

Thus $R_{p, q}$ is the finite dimensional vector space of $p$-th order syzygies of degree $q$ for $A$.

Example 3.3. Consider the Plücker embedding of the Grassmannian $G(2,5)$,

$$
\begin{aligned}
G(2,5) & \longrightarrow \mathbb{P}\left(\bigwedge^{2} \mathbb{C}^{5}\right) \\
\left\langle v_{1}, v_{2}\right\rangle_{\mathbb{C}} & \longmapsto\left[v_{1} \wedge v_{2}\right] .
\end{aligned}
$$

Let $\left\{e_{1}, \ldots, e_{5}\right\}$ be a basis of $\mathbb{C}^{5}$, then $G(2,5)$ can be written as the intersection of five quadrics $\Gamma_{i}=0$ where

$$
\begin{aligned}
& \Gamma_{1}=-e_{24} e_{35}+e_{23} e_{45}+e_{25} e_{34} \\
& \Gamma_{2}=-e_{14} e_{35}+e_{13} e_{45}+e_{15} e_{34} \\
& \Gamma_{3}=-e_{14} e_{25}+e_{12} e_{45}+e_{15} e_{24} \\
& \Gamma_{4}=-e_{13} e_{25}+e_{12} e_{35}+e_{15} e_{23} \\
& \Gamma_{5}=-e_{13} e_{24}+e_{12} e_{34}+e_{14} e_{23} .
\end{aligned}
$$

Here $e_{12}$ is the coordinate function dual to $e_{1} \wedge e_{2}$, etc. The projective coordinate algebra of $G(2,5)$ is $A=S(V) / I$ where $V$ is the vector space with basis $\left\{e_{i j}\right\}$ and $I=\left(\Gamma_{1}, \ldots, \Gamma_{5}\right)$.

From [8, Example 2.3.3] we know the dimensions of the syzygies and we can explicitly construct a minimal free resolution by $S(V)$ modules,

$$
\begin{aligned}
& 0 \longrightarrow F_{3} \longrightarrow F_{2} \longrightarrow F_{1} \longrightarrow F_{0} \longrightarrow A \longrightarrow \\
& \left\langle c^{*}\right\rangle \quad\left\langle\tilde{\Lambda}_{1}, \ldots, \tilde{\Lambda}_{5}\right\rangle \quad\left\langle\tilde{\Gamma}_{1}, \ldots, \tilde{\Gamma}_{5}\right\rangle \quad\langle c\rangle \quad S(V) / I
\end{aligned}
$$

In particular, $F_{0}=R_{0,0} \otimes S(V) \cong S(V)$ and the zeroth order syzygy $R_{0,0}$ is given by a copy of $\mathbb{C}$, generated by $c$. For exactness at $F_{0}$ we take $F_{1}=R_{1,2} \otimes S(V)$ where $R_{1,2}$ is spanned by $\tilde{\Gamma}_{1}, \ldots, \tilde{\Gamma}_{5}$ and $\partial \tilde{\Gamma}_{i}=\Gamma_{i} \cdot c$. The kernel of this boundary map is spanned by

$$
\begin{array}{rrrr}
\Lambda_{1} & = & -e_{12} \tilde{\Gamma}_{2}+e_{13} \tilde{\Gamma}_{3} & -e_{14} \tilde{\Gamma}_{4}+e_{15} \tilde{\Gamma}_{5} \\
\Lambda_{2} & =e_{12} \tilde{\Gamma}_{1} & -e_{23} \tilde{\Gamma}_{3} & +e_{24} \tilde{\Gamma}_{4}-e_{25} \tilde{\Gamma}_{5} \\
\Lambda_{3} & =-e_{13} \tilde{\Gamma}_{1} & +e_{23} \tilde{\Gamma}_{2} & -e_{34} \tilde{\Gamma}_{4}+e_{35} \tilde{\Gamma}_{5} \\
\Lambda_{4} & =e_{14} \tilde{\Gamma}_{1} & -e_{24} \tilde{\Gamma}_{2}+e_{34} \tilde{\Gamma}_{3} & -e_{45} \tilde{\Gamma}_{5} \\
\Lambda_{5} & =-e_{15} \tilde{\Gamma}_{1} & +e_{25} \tilde{\Gamma}_{2}-e_{35} \tilde{\Gamma}_{3} & +e_{45} \tilde{\Gamma}_{4}
\end{array}
$$


so for exactness at $F_{1}$ we take $F_{2}=R_{2,3} \otimes S(V)$ where $R_{2,3}$ is spanned by $\tilde{\Lambda}_{1}, \ldots, \tilde{\Lambda}_{5}$ and

$$
\begin{aligned}
& \partial \tilde{\Lambda}_{1}=\left(0,-e_{12}, e_{13},-e_{14}, e_{15}\right) \\
& \partial \tilde{\Lambda}_{2}=\left(e_{12}, 0,-e_{23}, e_{24},-e_{25}\right) \\
& \partial \tilde{\Lambda}_{3}=\left(-e_{13}, e_{23}, 0,-e_{34}, e_{35}\right) \\
& \partial \tilde{\Lambda}_{4}=\left(e_{14},-e_{24}, e_{34}, 0,-e_{45}\right) \\
& \partial \tilde{\Lambda}_{5}=\left(-e_{15}, e_{25},-e_{35}, e_{45}, 0\right)
\end{aligned}
$$

The third and highest order syzygy $R_{3,5}$ has a single generator $c^{*}$ with

$$
\partial c^{*}=\sum_{i=1}^{5} \Gamma_{i} \tilde{\Lambda}_{i}
$$

We return to this example in Example 4.4, where we write down the algebra structure on these syzygies.

3.2. The Berkovits complex. Let $A$ be a commutative quadratic algebra given by $S(V) / I$, where $V$ is a vector space with basis $a_{1}, \ldots, a_{n}$, and let $W_{1}$ be the vector space concentrated in degree 1 with basis $\theta_{1}, \ldots, \theta_{n}$.

Lemma 3.4. Suppose the quadratic ideal I is spanned by

$$
\Gamma_{k}=\sum_{i, j=1}^{n} \Gamma_{i j}^{k} a_{i} a_{j}, \quad(1 \leq k \leq m) .
$$

Then the first order syzygies are given by the following homology classes in $A\left[W_{1}\right]$,

$$
\tilde{\Gamma}_{k}=\sum_{i, j=1}^{n} \Gamma_{i j}^{k} a_{i} \theta_{j}, \quad(1 \leq k \leq m) .
$$

This leads to the following definition.

Definition 3.5. The Berkovits complex of a commutative quadratic algebra $A$ is

$$
A\left[W_{1} \oplus W_{2}\right]:=\left(A \otimes \bigwedge W_{1} \otimes S\left(W_{2}\right), \sum_{i=1}^{n} a_{i} \frac{\partial}{\partial \theta_{i}}+\sum_{k=1}^{m} \tilde{\Gamma}_{k} \frac{\partial}{\partial y_{k}}\right),
$$

where $W_{2}$ is the vector space spanned by elements $y_{1}, \ldots, y_{m}$ in homological degree two.

The Berkovits homology of $A$ is the homology of this complex.

Definitions 3.1 and 3.5, which calculate Koszul and Berkovits homology respectively, are examples of semi-free extensions of commutative DG algebras, or relative Sullivan algebras (see for example $[2,10,17,18]$ ):

Definition 3.6. $A$ semi-free extension of $A$ is an inclusion of commutative DG algebras

$$
A \rightarrow A \otimes S_{\bullet}(W)
$$

for some positively graded vector space $W$. We will write $A[W]$ for $A \otimes S_{\bullet}(W)$.

By $S \bullet$ we mean the symmetric algebra in the graded sense,

$$
S_{\bullet}(W)=S\left(W_{\text {even }}\right) \otimes \bigwedge W_{\text {odd }} .
$$

For instance Definition 3.5 would read $A\left[W_{1} \oplus W_{2}\right]=A \otimes S_{\bullet}\left(W_{1} \oplus W_{2}\right)$. 
3.3. Minimal models for commutative Koszul algebras. Assume that $A$ is a commutative Koszul algebra with $A^{!}=U(L)$ as above. We construct a resolution of $A$ in the category of DG algebras from the Chevalley-Eilenberg complex of $L$.

Definition 3.7. The Chevalley-Eilenberg complex of $L$ is the cochain complex with

$$
\mathrm{CE}^{i}(L)=\left(\bigwedge^{i} L\right)^{*}
$$

and the differential $d_{C}: \mathrm{CE}^{k}(L) \rightarrow \mathrm{CE}^{k+1}(L)$ is defined on $\varphi \in\left(\bigwedge^{k} L\right)^{*}$ by

$$
\left(d_{C} \varphi\right)\left(x_{0}, \ldots, x_{k}\right)=\sum_{i<j}(-1)^{j+\varepsilon(i, j)} \varphi\left(x_{0}, \ldots, x_{i-1},\left[x_{i}, x_{j}\right], x_{i+1}, \ldots, \widehat{x_{j}}, \ldots, x_{k}\right),
$$

where each $x_{r}$ is homogeneous, $\widehat{x_{j}}$ means omit $x_{j}$ and $\varepsilon(i, j)=\left|x_{j}\right|\left(\left|x_{i+1}\right|+\cdots+\left|x_{j-1}\right|\right)$.

The Chevalley-Eilenberg complex is a cochain complex that calculates the cohomology of $L$ with trivial coefficients, $H^{*}(L, \mathbb{C})$. Chevalley and Eilenberg in [5] introduced a shuffle product $\odot$ with respect to which the differential $d_{C}$ is a derivation and gives this complex an algebra structure that descends to the algebra structure on the cohomology of $L$.

For graded Lie superalgebras with finite dimensional graded components, one can prove that the shuffle product $\odot$ is skew-supersymmetric and that there is an algebra isomorphism

$$
\left(\bigwedge L^{*}, \wedge\right) \cong\left((\bigwedge L)^{*}, \odot\right)
$$

Hence, the Chevalley-Eilenberg complex can be alternatively defined as the exterior algebra on $L^{*}$, with differential $d_{C}$ the extension as a derivation of the map dual to $[]:, L \wedge L \rightarrow L$.

As well as being a cochain complex whose cohomology is that of $L$, the ChevalleyEilenberg complex may also be considered a chain complex which defines a resolution of $A$. The chain complex is given by defining $L_{p}^{*}$ to have homological grading $p-1$, so that the homological and cohomological gradings together give the total degree in $\bigwedge L^{*}$. We illustrate the Chevalley-Eilenberg complex as a chain complex with homological grading as follows:

$$
\begin{aligned}
& \cdots \mathrm{CE}_{4}(L) \stackrel{d_{C}}{\longrightarrow} \mathrm{CE}_{3}(L) \stackrel{d_{C}}{\longrightarrow} \mathrm{CE}_{2}(L) \stackrel{d_{C}}{\longrightarrow} \mathrm{CE}_{1}(L) \stackrel{d_{C}}{\longrightarrow} \mathrm{CE}_{0}(L) \\
& 0 \longrightarrow L_{1}^{*} \\
& 0 \longrightarrow L_{2}^{*} \longrightarrow \bigwedge^{2} L_{1}^{*} \\
& 0 \longrightarrow L_{3}^{*} \longrightarrow L_{2}^{*} \wedge L_{1}^{*} \longrightarrow \bigwedge^{3} L_{1}^{*} \\
& 0 \longrightarrow L_{4}^{*} \longrightarrow L_{3}^{*} \wedge L_{1}^{*} \oplus L_{2}^{*} \wedge L_{2}^{*} \longrightarrow L_{2}^{*} \wedge L_{1}^{*} \wedge L_{1}^{*} \longrightarrow \wedge^{4} L_{1}^{*}
\end{aligned}
$$

The original cohomological grading is seen on the diagonals: $\mathrm{CE}^{1}(L)=L^{*}$ is the first non-zero diagonal and $\mathrm{CE}^{2}(L)$ is the diagonal containing exterior products of two factors.

Definition 3.8. A minimal model of a commutative algebra $A=S(V) / I$ is a semi-free extension $S(V) \rightarrow S(V)[W]$ such that

(1) the canonical quotient $S(V)[W] \rightarrow S(V) \rightarrow A$ is an isomorphism in homology,

(2) the differential is decomposable, $\partial W \subseteq S(V) \otimes S_{\bullet}{ }^{2} W$.

This concept arose in rational homotopy theory, see for example [10, Definition 1.10] or [2, Section 7.2]. 
Proposition 3.9. For a commutative Koszul algebra $A$ with $A^{!}=U(L)$, the ChevalleyEilenberg complex of $L$ with homological grading is a minimal model of $A$.

Proof. We observe that $\mathrm{CE}_{0}(L)=\bigwedge L_{1}^{*}=S(V)$. If we take $W_{k}=L_{k+1}^{*}$ for $k \geq 1$ then $\mathrm{CE}(L)$ is the semi-free extension $S(V)[W]$.

As $L$ is graded, so is $\operatorname{Ext}_{U(L)}^{*}(\mathbb{C}, \mathbb{C})$, and since $A$ is Koszul

$$
H^{i}(L, \mathbb{C})_{j} \cong \operatorname{Ext}_{U(L)}^{i j}(\mathbb{C}, \mathbb{C})=\left\{\begin{array}{ll}
A & \text { if } i=j \\
0 & \text { if } i \neq j
\end{array},\right.
$$

where $j$ is the total degree. Using our homological grading of $\mathrm{CE}(L)$ we have therefore,

$$
H_{i}(\mathrm{CE}(L))= \begin{cases}A & \text { if } i=0 \\ 0 & \text { if } i \neq 0\end{cases}
$$

and the Chevalley-Eilenberg complex of $L$ is a resolution of $A$.

Finally, the differential is decomposable, since $d_{C}\left(L_{n}^{*}\right) \subseteq \sum_{i=1}^{n-2} L_{i}^{*} \wedge L_{n-i}^{*}$.

Remark 3.10. It follows that the Hilbert series of $A$ may also be calculated as the Euler characteristic of the Chevalley-Eilenberg complex with homological grading, and we recover (4).

We will now give a proof of the following theorem, based on a more general result of Avramov.

Theorem 3.11. The algebra of syzygies of $A$ is isomorphic to $H^{*}\left(L_{\geq 2}, \mathbb{C}\right)$ and the Berkovits homology is isomorphic to $H^{*}\left(L_{\geq 3}, \mathbb{C}\right)$.

Recall first the following definition from [2, Section 6.3].

Definition 3.12. The acyclic closure of a commutative DG algebra $A$ is a semi-free extension $A \rightarrow A[W]$ such that $A[W]$ is a resolution of $\mathbb{C}$ and $\left\{\partial w \mid w \in W_{n+1}\right\}$ minimally generates the reduced homology $\widetilde{H}_{n}\left(A\left[W_{\leq n}\right]\right)$ for each $n \geq 0$.

Thus the complexes calculating the Koszul and Berkovits homology are simply the first two steps of the acyclic closure of $A$. The following result of Avramov may be used to relate a general stage of the acyclic closure to the minimal model of $A$.

Recall that two DG algebras $X$ and $X^{\prime}$ are quasi-isomorphic if there is a sequence of quasi-isomorphisms of DG algebras $X \sim X^{1} \sim \cdots \sim X^{n} \sim X^{\prime}$ pointing in either direction.

Theorem 3.13 ([2, Theorem 7.2.6]). Suppose A is a commutative DG algebra with acyclic closure $A[W]$ and minimal model $S(V)\left[W^{\prime}\right]$. Then, for each $n \geq 1$, the $D G$ algebras $A\left[W_{\leq n}\right]$ and $S(V)\left[W^{\prime}\right] /\left(W_{<n}^{\prime}\right)$ are quasi-isomorphic.

If $A$ is Koszul with $A^{!}=U(L)$ then the Chevalley-Eilenberg complex of $L$ with homological grading is a minimal model $S(V)\left[W^{\prime}\right]$ of $A$, where $W_{i}^{\prime}=L_{i+1}^{*}$. Since $S(V)\left[W^{\prime}\right] /\left(W_{<n}^{\prime}\right)=\mathrm{CE}\left(L_{\geq n+1}\right)$ the theorem gives a quasi-isomorphism

$$
A\left[W_{\leq n}\right] \sim \mathrm{CE}\left(L_{\geq n+1}\right) .
$$

Theorem 3.11 is just the cases $n=1$ and 2 .

\section{A SMAller COMPleX}

Let $A$ be a Koszul commutative algebra, with Koszul dual $A^{!}$the universal enveloping algebra of a graded Lie superalgebra $L$. We have seen above that the Chevalley-Eilenberg complex of $L$ (with homological grading) is a resolution of $A$, and the Chevalley-Eilenberg 
where $V \cong \mathbb{C}^{N}$, the standard module for $\mathrm{GL}_{N}(\mathbb{C})$ or $\mathrm{SL}_{N}(\mathbb{C})$. We do not make these relations explicit, except to note that the semistandard Young tableaux in $\pi_{\left(\alpha_{1}, \ldots, \alpha_{p} \mid \beta_{1}, \ldots, \beta_{p}\right)}$ define basis vectors in this representation. For example the basis vector for (6) is

$$
\left(e_{1} \wedge e_{3} \wedge e_{4} \wedge e_{5} \wedge e_{6}\right) \otimes\left(e_{1} \wedge e_{3} \wedge e_{5} \wedge e_{6}\right) \otimes\left(e_{2} \wedge e_{4} \wedge e_{5} \wedge e_{6}\right) \otimes\left(e_{4} \wedge e_{6}\right) \otimes e_{5} \otimes e_{5},
$$

where $e_{1}, \ldots, e_{6}$ are a basis of the standard module for $\mathrm{GL}_{6}(\mathbb{C})$ or $\mathrm{SL}_{6}(\mathbb{C})$. Further, $\pi_{\left(\lambda_{1}, \ldots, \lambda_{N}\right)} \cong \pi_{\left(\lambda_{1}-\lambda_{N}, \ldots, \lambda_{N-1}-\lambda_{N}, 0\right)} \otimes \operatorname{det}^{\lambda_{N}}$.

If $\mathfrak{h}$ is the standard Cartan subalgebra of diagonal matrices in $\mathfrak{g l}_{N}=\operatorname{Lie}\left(\mathrm{GL}_{N}(\mathbb{C})\right)$, we let $\varepsilon_{1}, \ldots, \varepsilon_{N}$ be the standard basis of $\mathfrak{h}^{*}$. The highest weight of the irreducible representation corresponding to a partition $\lambda$ is $\left(\lambda_{1}-\lambda_{N}\right) \varepsilon_{1}+\cdots+\left(\lambda_{N-1}-\lambda_{N}\right) \varepsilon_{N-1}$. We also note that the fundamental weights of $\mathrm{GL}_{N}(\mathbb{C})$ are $\omega_{i}=\varepsilon_{1}+\cdots+\varepsilon_{i}$ for $i=1, \ldots, N-1$. The highest weight of the representation defined by Young diagrams of shape depicted in (6) is $6 \varepsilon_{1}+4 \varepsilon_{2}+3 \varepsilon_{3}+3 \varepsilon_{4}+\varepsilon_{5}$ or $[2,1,0,2,1]$ written in a basis of fundamental weights.

One introduces the algebra

$$
\mathrm{A}(N)=\bigoplus_{p, q} \mathrm{~A}_{p, q}(N)
$$

where

$$
\mathrm{A}_{p, q}(N):=\bigoplus_{\substack{N-2 \geq i_{1}>\cdots>i_{p} \geq 2 \\ i_{1}+\cdots+i_{p}=q}} \pi_{\left(\left(i_{1}-2\right), \ldots,\left(i_{p}-2\right) \mid\left(i_{1}+1\right), \ldots,\left(i_{p}+1\right)\right)} .
$$

This algebra is generated by

$$
\mathrm{A}_{1, r}=\pi_{(r-2 \mid r+1)}
$$

for $2 \leq r \leq N-2$. The multiplication is given by the projection onto the relevant irreducible component in the tensor product representation. We have the following:

Theorem 4.2. [8, Theorem 4.4.1] The algebra of syzygies of $G(2,5)$ form a bigraded supercommutative Frobenius Koszul algebra isomorphic to A(5) with

$$
\left(R_{G(2,5)}\right)_{-p+q, q}=\mathrm{A}_{p, q}(5) .
$$

Remark 4.3. The theorem as stated in [8] claims that the syzygies of $G(2, N)$ form a bigraded supercommutative Frobenius Koszul algebra. We suggest why this is not true for $N=6$. In particular the existence of a non-trivial ternary product implies that it is not Koszul.

Example 4.4. We return to Example 3.3 and consider the syzygies of $G(2,5)$, in which case

$$
\begin{array}{rcccccccc}
\mathrm{A}(5) & = & \mathrm{A}_{0,0}(5) & \oplus & \mathrm{A}_{1,2}(5) & \oplus & \mathrm{A}_{1,3}(5) & \oplus & \mathrm{A}_{2,5}(5) \\
& = & \pi_{\emptyset} & \oplus & \pi_{(0 \mid 3)} & \oplus & \pi_{(1 \mid 4)} & \oplus & \pi_{(10 \mid 43)} \\
& = & \mathbb{C} & \oplus & {[0,0,0,1]} & \oplus & {[1,0,0,0]} & \oplus & \mathbb{C} \\
& = & \left(R_{G(2,5)}\right)_{0,0} & \oplus & \left(R_{G(2,5)}\right)_{1,2} & \oplus & \left(R_{G(2,5)}\right)_{2,3} & \oplus & \left(R_{G(2,5)}\right)_{3,5},
\end{array}
$$

where $\pi_{\emptyset}$ denotes the empty semistandard Young tableau and the corresponding generator in $R_{G(2,5)}$ is given by $c$. The full list of other semistandard Young tableaux along with their generators in $R_{G(2,5)}$ are given by

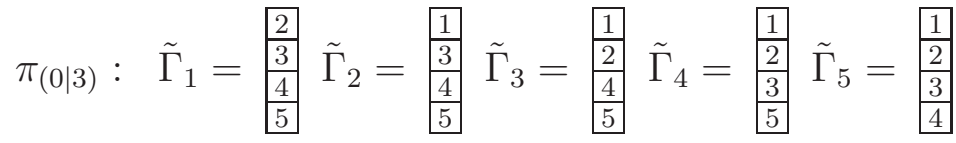

$$
\begin{aligned}
& \pi_{(1 \mid 4)}: \quad \tilde{\Lambda}_{i}=\begin{array}{|l|l|}
\hline \frac{1}{2} & i \\
\hline 3 & \\
\hline 4 & \\
\hline 5 &
\end{array} \quad \pi_{(10 \mid 43)}: c^{*}=\begin{array}{|l|l|}
\hline 1 & 1 \\
\hline 2 & 2 \\
\hline 3 & 3 \\
\hline 4 & 4 \\
\hline 5 & 5 \\
\hline
\end{array}
\end{aligned}
$$


so that $\operatorname{dim} \pi_{(0 \mid 3)}=\operatorname{dim} \pi_{(1 \mid 4)}=5$ and $\operatorname{dim} \pi_{(10 \mid 43)}=1$. Further, the algebra structure is given by $\tilde{\Gamma}_{i} \tilde{\Lambda}_{j}=\tilde{\Lambda}_{j} \tilde{\Gamma}_{i}=(-1)^{i+1} \delta_{i j} c^{*}, c$ is the unit of the algebra and all other multiplications are zero.

Example 4.5. We consider the syzygies of $G(2,6)$ given by $\mathrm{A}(6)=\mathrm{A}_{0}(6) \oplus \mathrm{A}_{1}(6) \oplus$ $\mathrm{A}_{2}(6) \oplus \mathrm{A}_{3}(6)$ where

$$
\begin{aligned}
& \mathrm{A}_{1}(6)=\mathrm{A}_{1,2}(6) \quad \oplus \quad \mathrm{A}_{1,3}(6) \quad \oplus \quad \mathrm{A}_{1,4}(6)
\end{aligned}
$$

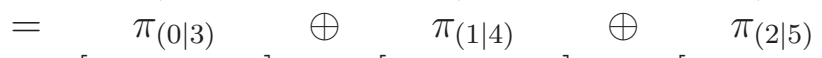

$$
\begin{aligned}
& =[0,0,0,1,0] \oplus[1,0,0,0,1] \oplus[2,0,0,0,0] \\
& =\left(R_{G(2,6)}\right)_{1,2} \oplus \quad\left(R_{G(2,6)}\right)_{2,3} \oplus \quad\left(R_{G(2,6)}\right)_{3,4}, \\
& \mathrm{~A}_{2}(6)=\mathrm{A}_{2,5}(6) \quad \oplus \quad \mathrm{A}_{2,6}(6) \quad \oplus \quad \mathrm{A}_{2,7}(6)
\end{aligned}
$$

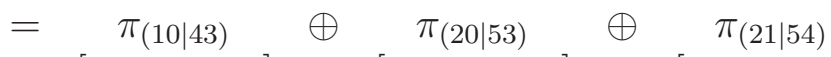

$$
\begin{aligned}
& =[0,0,0,0,2] \oplus[1,0,0,0,1] \oplus[0,1,0,0,0] \\
& =\left(R_{G(2,5)}\right)_{3,5} \oplus \quad\left(R_{G(2,6)}\right)_{4,6} \oplus \quad\left(R_{G(2,6)}\right)_{5,7},
\end{aligned}
$$

and $\mathrm{A}_{0}(6)=\mathrm{A}_{3}(6)=\mathbb{C}$.

We suggest two reasons why this is not a Koszul algebra. The first is that it is not a hook algebra in the sense of [8, Section 5.1]: it fails to satisfy $x \cdot y=0$ for all $x, y \in \pi_{T}$ where $T$ is a partition. Indeed by the Littlewood-Richardson rule $\pi_{(1 \mid 4)} \otimes \pi_{(1 \mid 4)} \supset \pi_{(20 \mid 53)}$ is not zero as claimed.

Further the syzygies appear to have a nontrivial $A_{\infty}$-algebra structure. Certainly one can see there is a ternary product $m_{3}: \mathrm{A}_{1,2}(6) \otimes \mathrm{A}_{1,2}(6) \otimes \mathrm{A}_{1,2}(6) \rightarrow \mathrm{A}_{2,6}(6)$ directly by multiplying the Schur functions ${ }^{1}$

$$
s_{(0 \mid 3)}{ }^{3}=s_{(10 \mid 54)}+2 s_{(20 \mid 53)}+3 s_{(21 \mid 52)}+s_{(21 \mid 43)}+s_{(210 \mid 510)}+2 s_{(210 \mid 420)}+s_{(210 \mid 321)} .
$$

The second factor is precisely $\mathrm{A}_{2,6}(6)$. If the algebra were Koszul then there could be no such nontrivial map of homological degree 1 and internal degree 0 .

4.2. The Complex. We now construct the complex that calculates the Berkovits homology for $A_{G(2,5)}$ with $A_{G(2,5)}^{!}=U(L)$. Initially, the idea was to construct this complex for a general commutative Koszul algebra whose algebra of syzygies is also Koszul. However, as pointed out to us by the referee, this is a rare situation in the real world. We borrow the name ' $b v_{\mu}$ ' from Movshev-Schwarz. Choose a basis $\left\{y_{1}, \ldots, y_{5}\right\}$ of $L_{2}$ inside the symmetric algebra $S\left(L_{2}\right)$ and consider the complex

$$
b v_{\mu}=S\left(L_{2}\right) \otimes_{\mathrm{A}(5) !} K\left(\mathrm{~A}(5)^{!}\right) \cong S\left(L_{2}\right) \otimes_{\mathrm{A}(5) !} \mathrm{A}(5)^{!} \otimes_{\mathbb{C}} \mathrm{A}(5)^{*} .
$$

Here $L_{2}$ is concentrated in degree 0 , and as usual for the Koszul complex we have $\mathrm{A}(5)^{\text {! }}$ concentrated in degree 0 and $\mathrm{A}(5)^{*}$ has grading induced by having its generators concentrated in degree 1 . In the more general situation, one would have to replace the Koszul resolution by a suitable resolution of the $A_{\infty}$-algebra of syzygies $R$, such as the bar resolution.

Consider the degree zero map of quadratic algebras

$$
\bigwedge L_{2}^{*} \rightarrow \mathrm{A}(5), \quad y_{k}^{*} \mapsto \tilde{\Gamma}_{k} .
$$

The dual map induces a map $\mathrm{A}(5)^{!} \rightarrow S\left(L_{2}\right)$ which gives an action of $\mathrm{A}(5)^{!}$on $S\left(L_{2}\right)$ by multiplication. Again, in the more general situation we would have to consider $S\left(L_{2}\right)$ as an $R$-module and this action may be more complicated.

\footnotetext{
${ }^{1}$ The calculation was performed using SAGE.
} 
The differential is $1 \otimes d$ where $d$ is the Koszul differential of $K\left(\mathrm{~A}(5)^{!}\right)$. Thus we may write

$$
b v_{\mu}=\left(S\left(L_{2}\right) \otimes \mathrm{A}(5)^{*}, \quad \sum_{k=1}^{5} y_{k} \frac{\partial}{\partial \tilde{\Gamma}_{k}^{*}}\right) .
$$

In more general cases the differential would have a more complicated form due to the presence of higher multiplications and may contain terms of higher order in $\partial / \partial \tilde{\Gamma}_{k}^{*}$.

Taking into account the natural grading on the symmetric algebra we see that $b v_{\mu}$ is filtered by the complexes $S^{\geq p}\left(L_{2}\right) \otimes \mathrm{A}(5)^{*}$ and the associated graded is isomorphic to the bigraded vector space

$$
\operatorname{Gr}\left(b v_{\mu}\right)=S\left(L_{2}\right) \otimes \mathrm{A}(5)^{*} .
$$

In the more general case there is no guarantee that this associated graded object has no differential.

Choose a basis $\left\{q_{1}, \ldots, q_{m}\right\}$ of $L_{2}$ inside $U\left(L_{\geq 2}\right)$. Now let

$$
E_{\mu}=\left(\bigwedge L_{2} \otimes U\left(L_{\geq 2}\right), \sum_{k=1}^{m} y_{k} \frac{\partial}{\partial q_{k}}\right)
$$

with the standard augmented algebra structure. This has a filtration $\bigwedge^{\geq p} L_{2} \otimes U\left(L_{\geq 2}\right)$ whose associated graded $\operatorname{Gr}\left(E_{\mu}\right)$ is the algebra itself but with zero differential. The reduced bar complex $B^{+} E_{\mu}$ has an induced filtration, and we observe that the corresponding associated graded complex is isomorphic to the bar complex of the associated graded,

$$
\operatorname{Gr}\left(B^{+} E_{\mu}\right)=B^{+}\left(\operatorname{Gr}\left(E_{\mu}\right)\right)=B^{+}\left(\bigwedge L_{2} \otimes U\left(L_{\geq 2}\right)\right) .
$$

We show that $b v_{\mu}$ calculates the cohomology of $L_{\geq 3}$ by adapting the argument from [11, Section 3] which studies the case where $R^{!} \cong U\left(L_{\geq 2}\right)$ is the Yang-Mills algebra, and which is also inspired by [15].

The idea is to use the complex $B^{+} E_{\mu}$ as an intermediary whose homology is the same as $b v_{\mu}$ and $\mathrm{CE}\left(L_{\geq 3}\right)$ :

$$
b v_{\mu} \stackrel{\sim}{\longrightarrow} B^{+} E_{\mu} \stackrel{\sim}{\longleftarrow} \mathrm{CE}\left(L_{\geq 3}\right)
$$

The map $p: b v_{\mu} \rightarrow B^{+} E_{\mu}$ is constructed as the composition of a map $b v_{\mu} \rightarrow E_{\mu}$ with the quasi-isomorphism $E_{\mu} \rightarrow B^{+} E_{\mu}$. If we map $y_{k} \mapsto y_{k}, \tilde{\Gamma}_{k} \mapsto q_{k}$ and $\tilde{\Lambda}_{k}$ to the corresponding generator in $L_{3}$ inside $U\left(L_{\geq 2}\right)$, then this map clearly respects the filtrations.

Theorem 4.6. Homologies of the complexes $b v_{\mu}$ and $B^{+} E_{\mu}$ coincide.

Proof. Consider the spectral sequences associated to the filtrations on $b v_{\mu}$ and $B^{+} E_{\mu}$ defined above, which converge to their respective homologies and which have zero pages the associated graded objects (7), (8).

By Theorem 3.11 we can say that there exists a chain map

$$
\mathrm{A}(5)^{*} \cong \mathrm{A}(5) \stackrel{\sim}{\longrightarrow} \mathrm{CE}\left(L_{\geq 2}\right) \stackrel{\sim}{\longrightarrow} B^{+}\left(U\left(L_{\geq 2}\right)\right)
$$

that induces isomorphism of homology groups, given by some choice of homology classes in the Chevalley-Eilenberg or bar complexes. Using this, together with classical Koszul duality for symmetric and exterior algebras, and the Künneth theorem, we can construct a chain map

$$
S\left(L_{2}\right) \otimes \mathrm{A}(5)^{*} \stackrel{\sim}{\longrightarrow} B^{+}\left(\bigwedge L_{2}\right) \otimes B^{+}\left(U\left(L_{\geq 2}\right)\right) \stackrel{\sim}{\longrightarrow} B^{+}\left(\bigwedge L_{2} \otimes U\left(L_{\geq 2}\right)\right)
$$

inducing isomorphism in homology. In other words, we have a chain map inducing isomorphism between the homologies of the associated graded objects above,

$$
\operatorname{Gr}\left(b v_{\mu}\right) \stackrel{\sim}{\longrightarrow} \operatorname{Gr}\left(B^{+} E_{\mu}\right) .
$$


This is a weak equivalence between the zero pages of the spectral sequences associated to the filtrations on $b v_{\mu}$ and $B^{+} E_{\mu}$. Hence we have an isomorphism between the first pages. The result follows from the comparison theorem of spectral sequences.

For the second quasi-isomorphism we adapt the proof in [11].

Proposition 4.7. The algebras $E_{\mu}$ and $U\left(L_{\geq 3}\right)$ are quasi-isomorphic, or the homologies of $B^{+}\left(E_{\mu}\right)$ and $\mathrm{CE}\left(L_{\geq 3}\right)$ coincide. The quasi-isomorphism is given by the inclusion $z \in$ $U\left(L_{\geq 3}\right) \mapsto 1 \otimes z \in E_{\mu}$.

Proof. We must prove that the cohomology of $E_{\mu}$ is $U\left(L_{\geq 3}\right)$. Consider the following filtration of $U\left(L_{\geq 2}\right)$ :

$$
F^{j}:=\left\{\begin{array}{cc}
0 & j=0 \\
\left\{z \in U\left(L_{\geq 2}\right) \mid \frac{\partial}{\partial q_{i}}(z) \in F^{j-1} \forall i\right\} & j>0
\end{array}\right.
$$

Poincaré-Birkhoff-Witt implies that $F^{1}=U\left(L_{\geq 3}\right.$ ) (see [11, Proposition 3.1] for more details) and by [15, Lemma 28] the filtration is multiplicative, exhaustive, Hausdorff $\left(\bigcap F^{j}=0\right)$ and $q_{i} \in F^{2}$. We define a filtration of $E_{\mu}$ as in [11, Proposition 3.7]:

$$
F_{p} E_{\mu, q}:=F^{p+5-q} \otimes \bigwedge^{5-q} L_{2} .
$$

It is clear that $d\left(F_{p} E_{\mu, q}\right) \subseteq F_{p-2} E_{\mu, q-1}$, where $d$ is the differential on the complex $E_{\mu}$, and so the differentials on the $E^{0}$ and $E^{1}$-pages of the spectral sequence associated to this filtration are zero.

Since [15, Lemma 29] is true in this context, we have that $\operatorname{Gr}_{F}\left(U\left(L_{\geq 2}\right)\right) \cong U\left(L_{\geq 3}\right) \otimes$ $\mathbb{C}\left[\hat{q}_{1}, \ldots, \hat{q}_{5}\right]$, where $\hat{q}_{1}, \ldots, \hat{q}_{5}$ are the images of $q_{1}, \ldots, q_{5}$ in $\operatorname{Gr}_{F}\left(U\left(L_{\geq 2}\right)\right)$. The $E^{2}$-page is given by

$$
E_{p q}^{2}=F_{p} E_{\mu, p+q} / F_{p-1} E_{\mu, p+q} \cong U\left(L_{\geq 3}\right) \otimes \mathbb{C}\left[\hat{q}_{1}, \ldots, \hat{q}_{5}\right] \otimes \bigwedge^{5-p-q} L_{2} .
$$

The differential on the $E^{2}$-page is nothing but the Koszul differential for $\bigwedge L_{2}$ (see [11, Proposition 3.7] for more details), which is a resolution of $\mathbb{C}$. Hence, the spectral sequence collapses on the $E^{3}$-page to $E_{0,5}^{3}=U\left(L_{\geq 3}\right)$.

We have the following immediate corollary.

Corollary 4.8. The complex bv $v_{\mu}$ calculates the Berkovits homology. Further, we have $H^{2}\left(L_{\geq 3}, \mathbb{C}\right)=0$ and so $L_{\geq 3}$ is free.

Proof. The first statement is clear. For the second statement, we use a result from [4, Ex II.2.9] that if $L_{\geq 3}$ is positively graded then $L_{\geq 3}$ is a free Lie (super)algebra if and only if $H^{2}\left(L_{\geq 3}, \mathbb{C}\right)=0$. The differential in the complex $b v_{\mu}$ is:

$$
d(c)=0, \quad d\left(\tilde{\Gamma}_{i}\right)=y_{i} c, \quad d\left(\tilde{\Lambda}_{i}\right)=0, \quad d\left(c^{*}\right)=\sum_{i=1}^{5}(-1)^{i+1} y_{i} \tilde{\Lambda}_{i} .
$$

Since the only contribution to $H^{2}\left(L_{\geq 3}, \mathbb{C}\right)$ could be given by $c^{*}$, and this is not a cycle, we obtain $H^{2}\left(L_{\geq 3}, \mathbb{C}\right)=0$.

4.3. Further generalization. We presented a simple construction of a complex $b v_{\mu}$ quasi-isomorphic to the Berkovits homology and to the Lie algebra $L_{\geq 3}$, which made certain calculations very straightforward. Although it is only constructed for $G(2,5)$, we would like to investigate whether the construction may be further extended to cases where the algebra of syzygies has higher structure.

We suspect that one may enrich the homology isomorphism between the complexes $b v_{\mu}$ and $B^{+} E_{\mu}$. Namely, one would like quasi-isomorphisms of differential graded algebras: 
between the cobar construction $\Omega b v_{\mu}$, the algebra $E_{\mu}$, and the algebra $U\left(L_{\geq 3}\right)$. For this, however, it would be necessary to define an appropriate coalgebra structure on $b v_{\mu}$. In the case that the algebra of syzygies $R$ is quadratic, $b v_{\mu}$ will will be a 'strict' differential graded coalgebra, and other cases it will be an $A_{\infty}$-coalgebra. An explicit definition of such an $A_{\infty}$ structure was given in the paper [15] of Movshev-Schwarz for the example they considered.

\section{REFERENCES}

[1] Avramov, L.L.; Free Lie subalgebras of the cohomology of local rings Trans. Amer. Math. Soc. 270 (1982), no. 2, 589-608.

[2] Avramov, L.L.; Infinite free resolutions. Six lectures on commutative algebra Progr. Math., 166, 1118, Birkhäuser, Basel, 1998.

[3] Berkovits, N.; Cohomology in the pure spinor formalism for the superstring, J. High Energy Phys. 9 (2000).

[4] Bourbaki, N.; Lie Groups and Lie Algebras, Chapters 1-3, Springer, 1991.

[5] Chevalley, C.; Eilenberg, S.; Cohomology Theory of Lie Groups and Lie Algebras Trans. Amer. Math. Soc. 63 (1948) 85-124.

[6] Eisenbud, D.; Commutative Algebra with a View Toward Algebraic Geometry, Grad. Text. in Math. Springer-Verlag, 1995. xvi+785 pp.

[7] Gauss C.; Disquisitiones generales de congruentis, Analysis residuorum. Caput octavum, Collected Works, Vol. 2, Georg Olms Verlag, Hildersheim, New York, 1973, 212-242.

[8] Gorodentsev, A.; Khoroshkin, A.; Rudakov A.; On syzygies of highest weight orbits, Amer. Math. Soc. Transl. 221 (2007).

[9] Gross, B. H.; Wallach, N. R.; On the Hilbert polynomials and Hilbert series of homogeneous projective varieties, In: Arithmetic geometry and automorphic forms, Adv. Lect. Math. 19 (2011) 253-263.

[10] Hess, K; Rational homotopy theory: a brief introduction. Interactions between homotopy theory and algebra, 175-202, Contemp. Math., 436, Amer. Math. Soc., Providence, RI, 2007.

[11] Herscovich, E.; Solotar, A.; Representations of Yang-Mills algebras, Annals of Mathematics 173 (2011), 1043-1080

[12] Kadeishvili, T.; The algebraic structure in the homology of an $A(\infty)$-algebra (Russian), Soobshch. Akad. Nauk Gruzin. SSR 108 (1982) 249-252

[13] Kang, S.-J.; Graded Lie Superalgebras and the Superdimension Formula, Journal of Algebra 204 (1998), 597-655

[14] MacLane, S.; Homology, Reprint of the 1975 edition, Springer-Verlag, Berlin, 1995. x+422 pp.

[15] Movshev, M.; Schwarz, A.; Algebraic structure of Yang-Mills theory, in The Unity of Mathematics: In Honor of the Ninetieth Birthday of I.M. Gelfand (Progress in Mathematics 244) Birkhuser (2006) 473-523.

[16] Polishchuk, A.; Positselski, L.; Quadratic algebras, University Lecture Series, 37. AMS, (2005). xii+159 pp.

[17] Quillen, D. Rational homotopy theory, Ann. of Math. (2) 90 (1969) 205-295.

[18] Sullivan, D.; Infinitesimal computations in topology, Publications Mathématiques de l'IHÉS, 47 (1977) 269-331.

Departament de Matemàtica Aplicada III, Universitat Politècnica de Catalunya, Escola d'Enginyeria de Terrassa, Carrer Colom 1, 08222 Terrassa (Barcelona), Spain

E-mail address: m.immaculada.galvez@upc.edu

Institute of Mathematics, University of Aberdeen, Fraser Noble Building, King's College, Aberdeen AB24 3UE, United Kingdom

E-mail address: vgorb@maths.abdn.ac.uk

FAKUltät FÜr Elektrotechnik, InFormatik und Mathematik, Institut Für Mathematik, Warburger Str. 100, 33098 Paderborn

E-mail address: zain@math.upb.de

Department of Mathematics, University of Leicester, University Road, Leicester LE1 7RH, United Kingdom

E-mail address: apt12@leicester.ac.uk 